Supporting Information

\title{
Superbase ionic liquid-based deep eutectic solvents for improving $\mathrm{CO}_{2}$ absorption
}

Hong Yan ${ }^{\dagger}$, Lei Zhao ${ }^{+,}$, Yinge Bai , Fangfang Li $i^{\ddagger}$, Haifeng Dong ${ }^{\ddagger}$, Hui Wang, Xiangping Zhang ${ }^{\ddagger, \S}$, Shaojuan Zeng*,*

${ }^{+}$College of Chemical and Environmental Engineering Harbin, University of Science and Technology No. 4, Linyuan Road, Harbin 150040 (China)

₹ Key Laboratory of Green Process and Engineering, State Key Laboratory of Multiphase Complex System, Beijing Key Laboratory of Ionic Liquids Clean Process, Institute of Process Engineering, Chinese Academy of Sciences, Beijing, 100190, China ${ }^{\S}$ College of Chemical and Engineering, University of Chinese Academy of Sciences, Beijing, 100049, China

Corresponding author.

E-mail address: sjzeng@ipe.ac.cn (S. J. Zeng); Tel./fax: +86-010-82544875

Number of pages: 9 (S1-S9)

Number of figures: 6 (Figure S1-S6)

Number of tables: 1 (Table S1) 


\section{Contents}



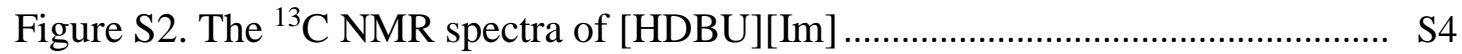

Figure S3. The ${ }^{1} \mathrm{H}$ NMR spectra of [HDBU][Ind] ................................................ S5

Figure S4. The ${ }^{13} \mathrm{C}$ NMR spectra of [HDBU][Ind] ................................................. S6

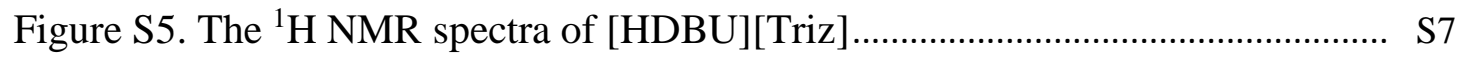

Figure S6. The ${ }^{13} \mathrm{C}$ NMR spectra of [HDBU][Triz].............................................. S8

Table S1. Viscosities of IL-based DESs before and after $\mathrm{CO}_{2}$ absorption $\quad . . \ldots \ldots \ldots \ldots . . . . \quad$ S9 

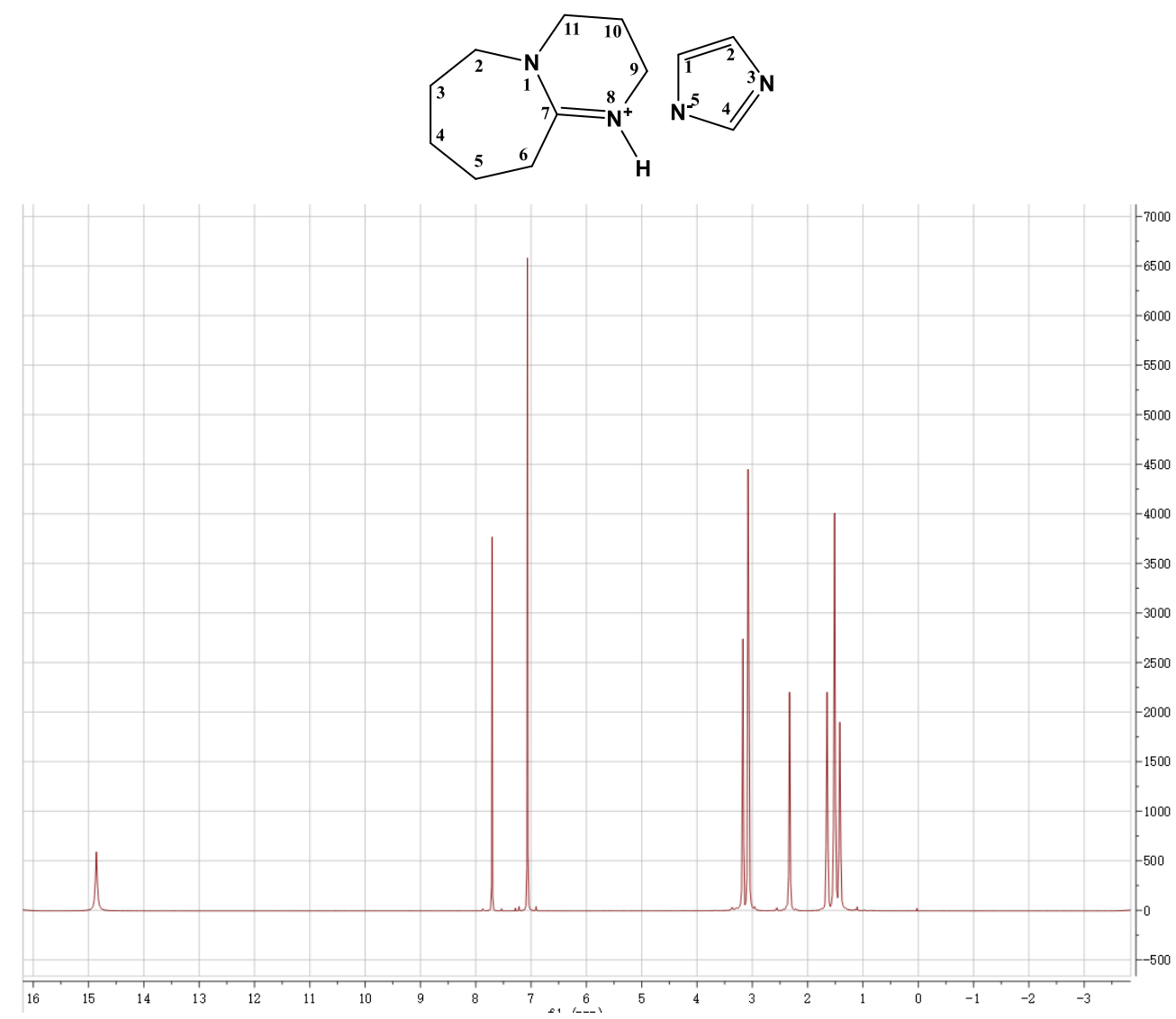

Figure S1. ${ }^{1} \mathrm{H}$ NMR spectra of [HDBU][Im]

${ }^{1} \mathbf{H}$ NMR (CDCl3): 1.42 (m, 2H, HDBU C4), 1.51 (m, 4H, HDBU C3 and C5), 1.66 (m, 2H, HDBU C10), 2.33 (t, 2H, HDBU C6), 3.09 (m, 4H, HDBU C2 and C11), 3.17 (t, 2H, HDBU C9), 7.07 (s, 2H, Im C4 and C5), 7.70 (s, 1H, Im C2), 14.86 (s, 1H, HDBU N8); 


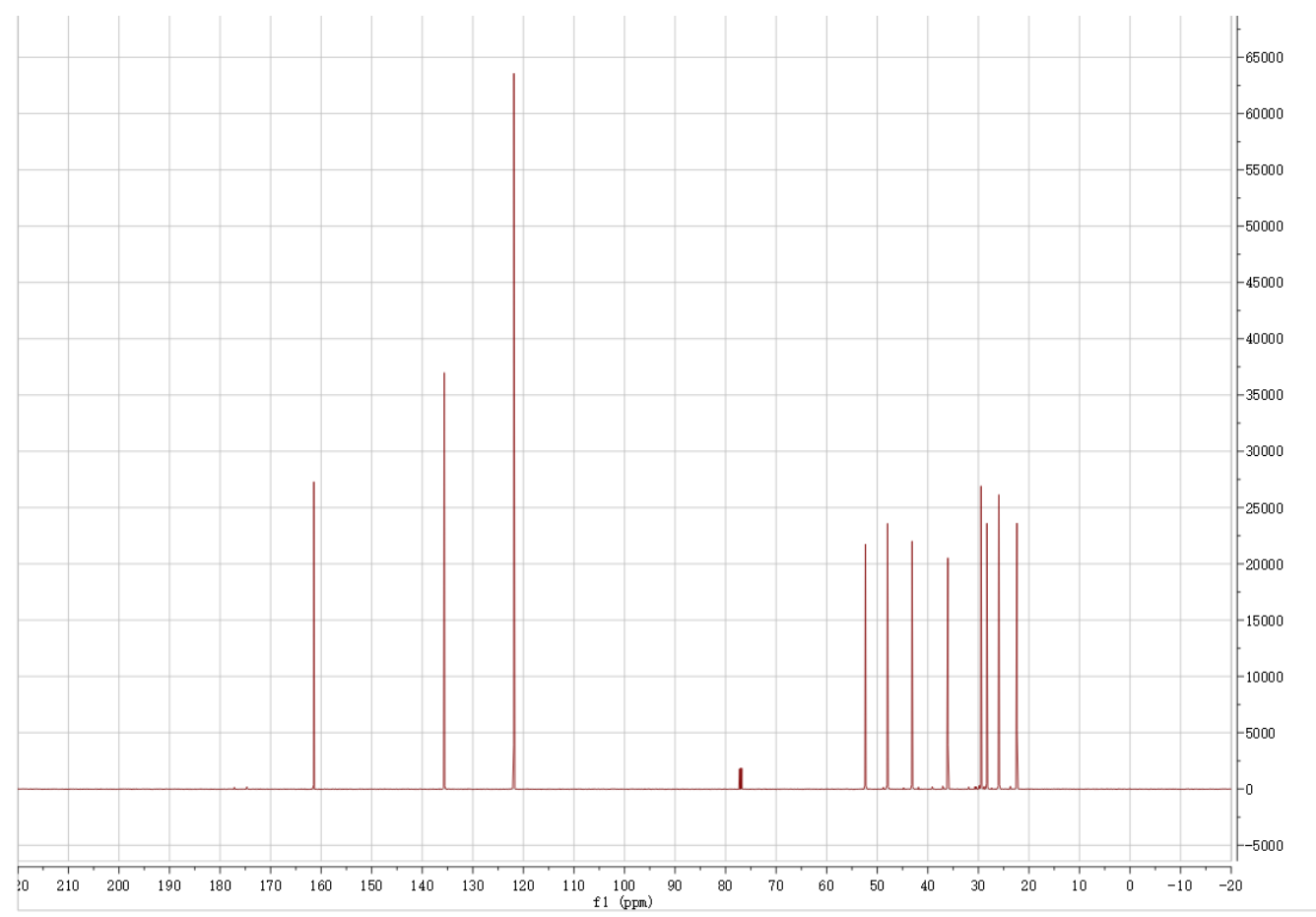

Figure S2. ${ }^{13} \mathrm{C}$ NMR spectra of [HDBU][Im]

${ }^{13}$ C NMR (CDCl3): 22.37 (HDBU C10), 25.95 (HDBU C4), 28.31 (HDBU C5), 29.47 (HDBU C3), 36.04 (HDBU C6), 43.11 (HDBU C9), 47.96 (HDBU C11), 52.34 (HDBU C2), 121.87 (Im C4 and C5), 135.65 (Im C2), 161.44 (HDBU C7) 

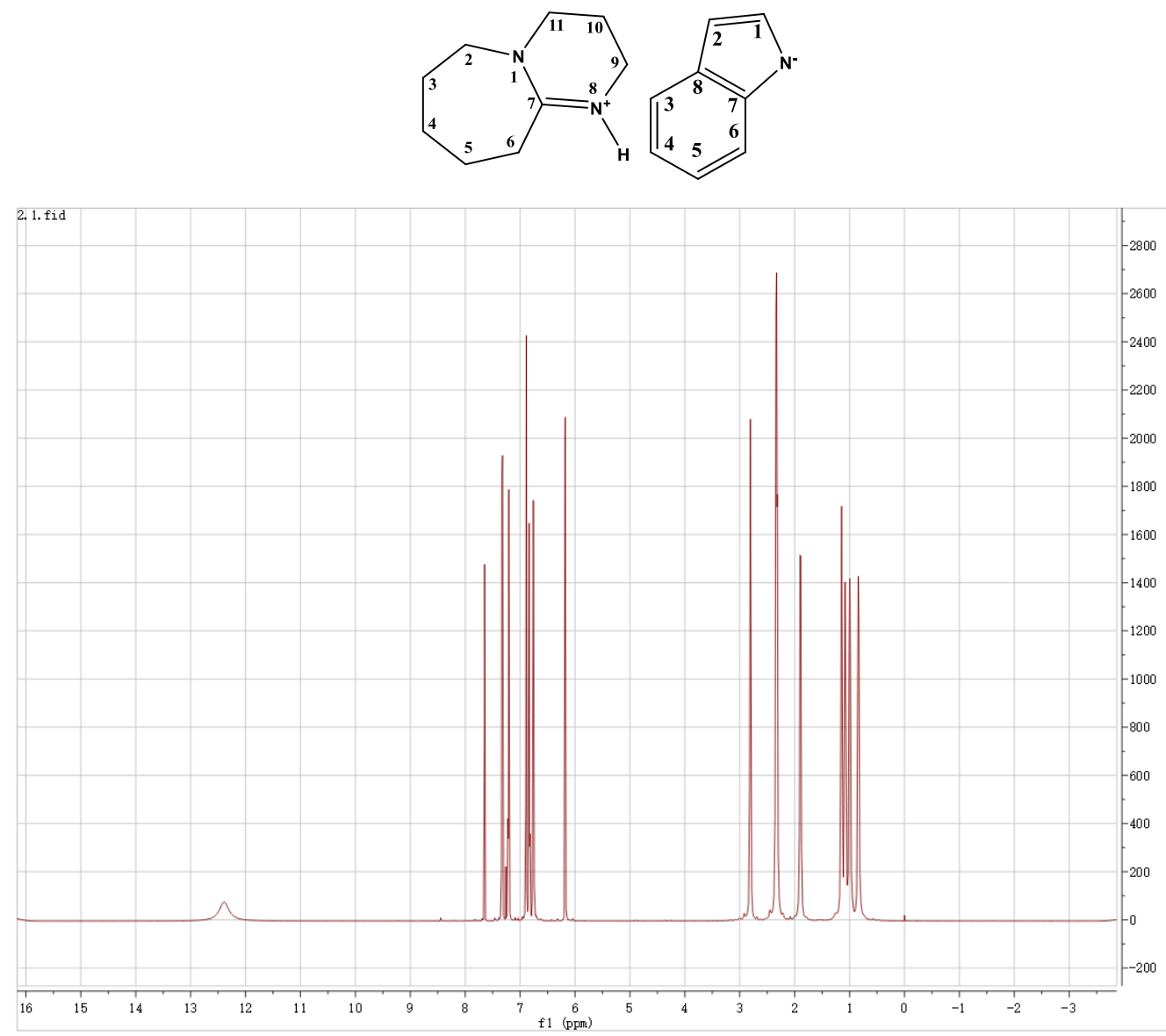

Figure S3. ${ }^{1} \mathrm{H}$ NMR spectra of [HDBU][Ind]

${ }^{1} \mathbf{H}$ NMR (CDCl3): 0.84 (m, 2H, HDBU C4), 1.00 1.08 (m, 4H, HDBU C3 and C5), 1.15 (m, 2H, HDBU C10), 1.89 (t, 2H, HDBU C6), 2.32 (m, 4H, HDBU C2 and C11), 2.80 (t, 2H, HDBU C9), 6.18 (d, 1H, Ind C1), 6.76 (t, 1H, Ind C5), 6.83 (t, 1H, Ind C4), 6.89 (d, Ind C2), 7.21 (d, 1H, Ind C6), 7.33 (d, 1H, Ind C3), 12.40 (s, 1H, HDBU N8); 


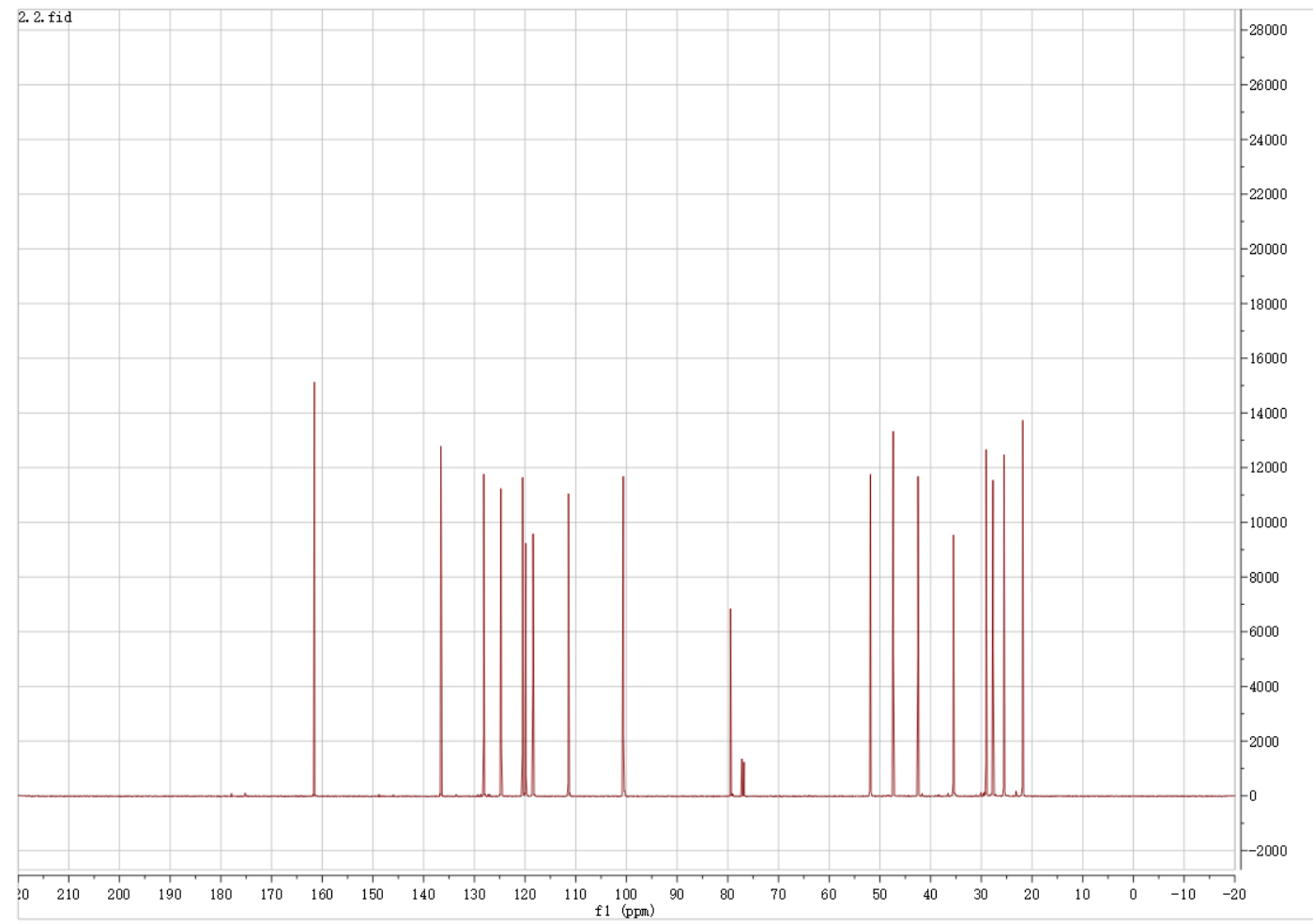

Figure S4. ${ }^{13} \mathrm{C}$ NMR spectra of [HDBU][Ind]

${ }^{13}$ C NMR (CDCl3): 22.46 (HDBU C10), 25.51 (HDBU C4), 28.51 (HDBU C5), 29.75 (HDBU C3), 37.17 (HDBU C6), 43.95 (HDBU C9), 48.39 (HDBU C11), 52.88 (HDBU C2), 102.17 (Ind C6), 111.05 (Ind C5), 119.53 (Ind C1), 120.61 (Ind C3), 121.70 (Ind C4), 124.13 (Ind C8), 127.85 (Ind C2), 135.78 (Ind C7), 161.79 (HDBU C7) 

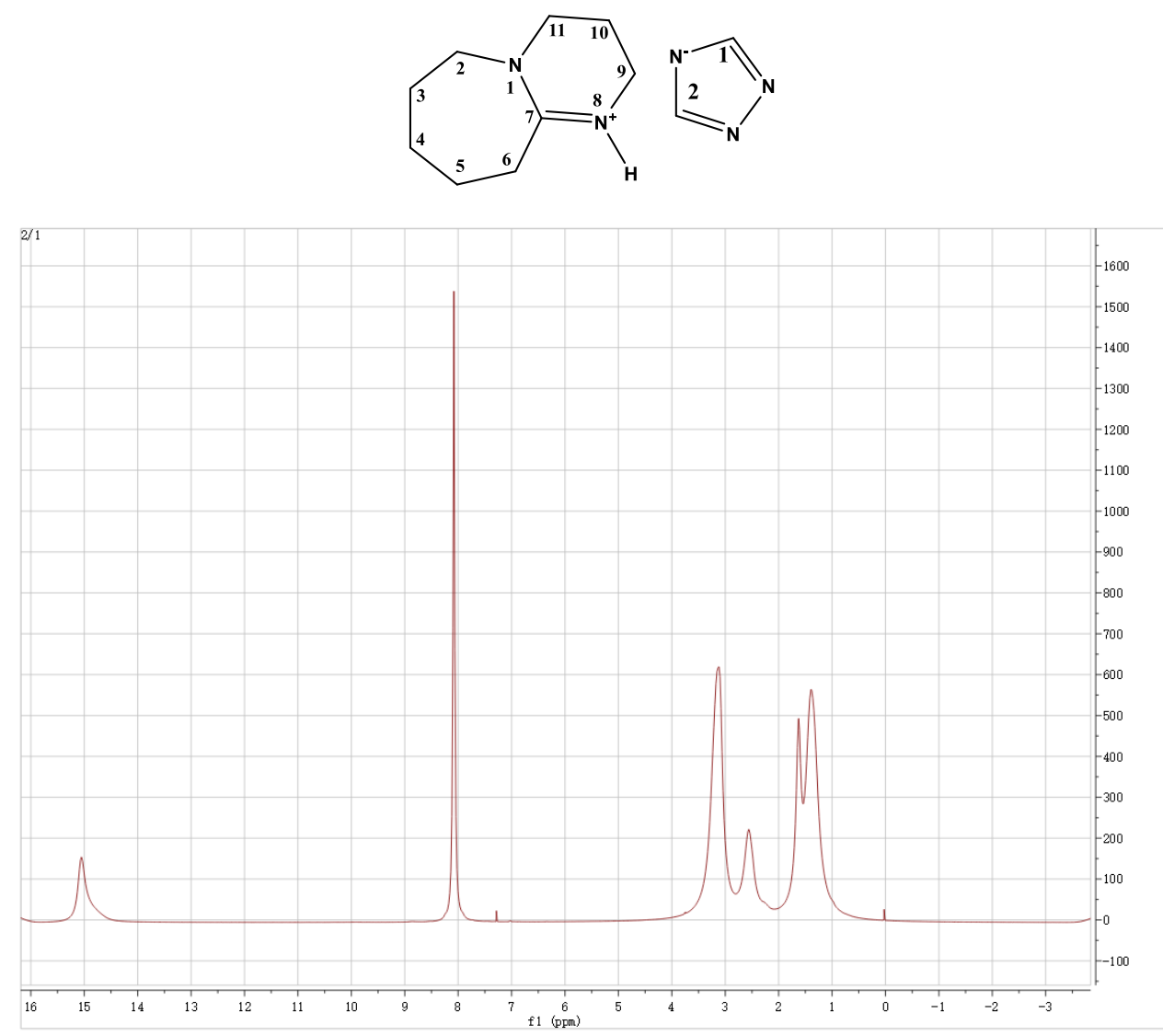

Figure S5. ${ }^{1} \mathrm{H}$ NMR spectra of [HDBU][Triz]

${ }^{1} \mathbf{H}$ NMR (CDCl3): 1.36 (m, 2H, HDBU C4), 1.36 (m, 4H, HDBU C3 and C5), 1.56 (m, 2H, HDBU C10), 2.46 (t, 2H, HDBU C6), 3.03 3.09 (m, 4H, HDBU C2 and C11), 3.03 3.09 (t, 2H, HDBU C9), 7.98 (s, 2H, Triz C1 and C2), 14.83 (s, 1H, HDBU N8); 


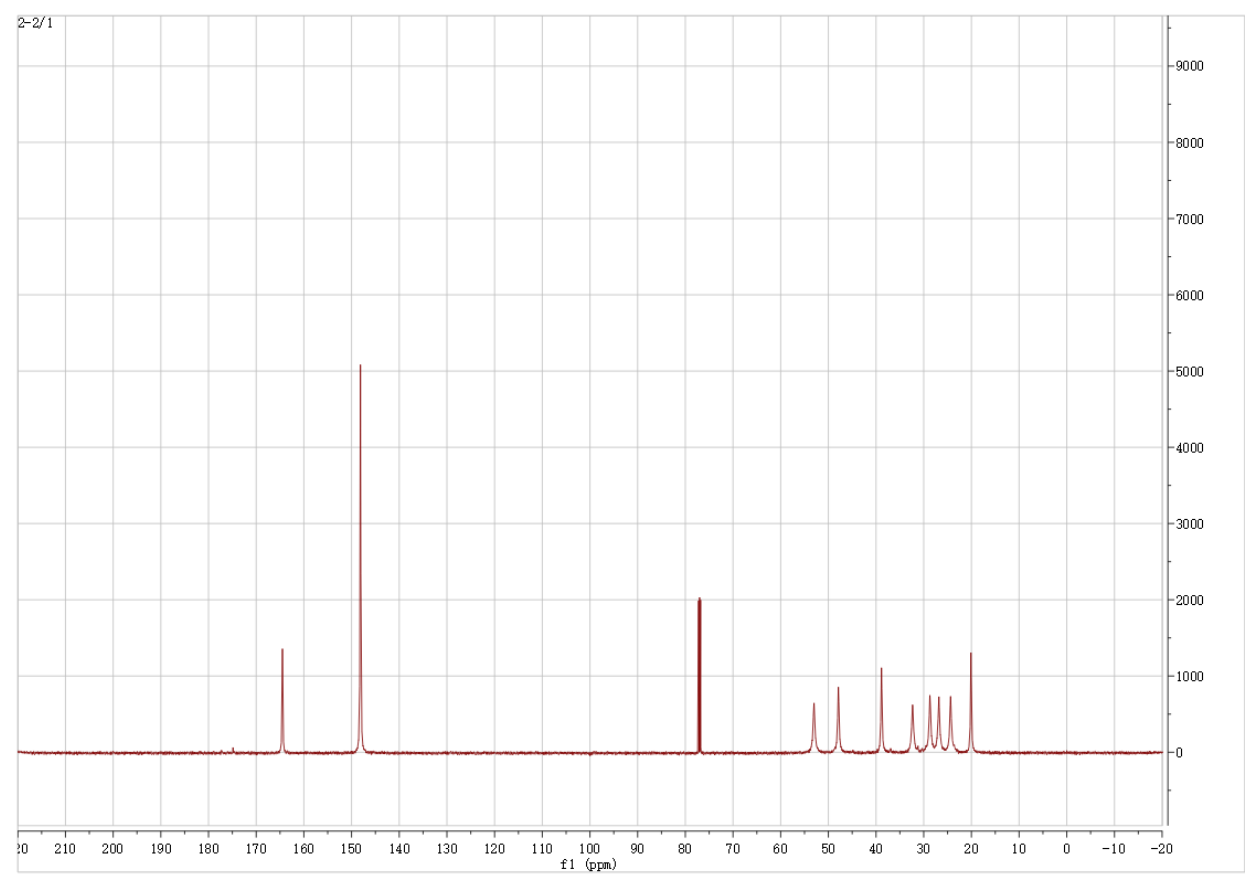

Figure S6. ${ }^{13} \mathrm{C}$ NMR spectra of [HDBU][Triz]

${ }^{13}$ C NMR (CDCl3): 19.40 (HDBU C10), 23.60 (HDBU C4), 26.04 (HDBU C5), 27.79 (HDBU C3), 31.78 (HDBU C6), 38.41 (HDBU C9), 46.91 (HDBU C11), 51.91 (HDBU C2), 75.98 (Triz C1), 147.13 (Triz C2), 163.06 (HDBU C7) 
Table S1. Viscosities of IL-based DESs before and after $\mathrm{CO}_{2}$ absorption at $40{ }^{\circ} \mathrm{C}$

IL-based DESs Viscosities before absorption Viscosities after absorption
(Mass ratio of 7:3)
$(\mathrm{mPa} \cdot \mathrm{s})$
$(\mathrm{mPa} \cdot \mathrm{s})$

$[\mathrm{HDBU}][\mathrm{Im}] / \mathrm{EG}$

31.48

166.51

[HDBU][Ind]/EG

36.99

114.18

[HDBU][Triz]/EG

197.72

205.26 